\title{
Análise da aplicação de Sistemas de Recomendação no contexto educacional: Revisão Sistemática da Literatura Nacional
}

Tamires Beatriz da Silva Lucena, ICT/Unifesp, tlucena@unifesp.br, https://orcid.org/0000-0002-4848-1814

Pedro Spoljaric Gomes, ICT/Unifesp, pedro.spoljaric@unifesp.br, https://orcid.org/0000-0003-4048-1366

Daniela Leal Musa, ICT/Unifesp, musa@unifesp.br, https://orcid.org/0000-0002-8405-959X

Tiago de Oliveira, ICT/Unifesp, tiago.oliveira@unifesp.br, https://orcid.org/0000-0002-3676-5967

Resumo: Os sistemas de recomendação, apresentados como uma possibilidade de personalização de informações, podem funcionar no contexto educacional para a construção do processo de aprendizagem e formação de usuários, visando o envio de dados (materiais, tipos de conteúdo e estratégias de aprendizagem) que são de interesse. Este trabalho teve como objetivo a construção de uma visão geral dos estudos nacionais que tratam dessa implementação, e também da validação e aplicação de sistemas de recomendação no âmbito educacional nos últimos 7 anos. A análise da revisão sistemática mostrou o modelo Felder-Silverman Learning Styles (FSLSM) e o padrão de metadados IEEE-LOM como as tecnologias mais utilizadas, bem como as técnicas de validação de projetos mais frequentes e outros aspectos gerais do planejamento e do desenvolvimento de sistemas. Os resultados apresentados apontam que, apesar da carência de estudos específicos acerca do uso de sistemas de recomendação para cursos de graduação, a relevância de recursos que melhorem a experiência acadêmica e facilitem o processo de aprendizagem recebe cada vez mais destaque.

Palavras-chave: recomendação, sistemas, educação, ensino, aplicação.

\section{Analysis of the application of Recommendation Systems in the educational context: Systematic Review of National Literature}

Abstract: Recommendation systems, presented as a possibility of personalization of information, can work in the educational context for the construction of the learning process and training of users, aiming at sending data (materials, types of content and learning strategies) that are of interest. This work aimed to build an overview of the national studies that deal with this implementation, as well as the validation and application of recommendation systems in the educational field in the last 7 years. The analysis of the systematic review showed the Felder-Silverman Learning Styles (FSLSM) model and the IEEE-LOM metadata standard as the most used technologies, as well as the most frequent project validation techniques and other general aspects of planning and development of projects. systems. The results presented show that, despite the lack of specific studies on the use of recommendation systems for undergraduate courses, the relevance of resources that improve the academic experience and facilitate the learning process is increasingly highlighted.

Keywords: recommendation, systems, education, teaching, application.

\section{Introdução}

Considerar diversos aspectos e personalizar o conteúdo a ser apresentado para o usuário é a proposta dos sistemas de recomendação, os quais vêm sendo utilizados, inclusive, na área da educação como um instrumento para o processo de aprendizagem e formação de cada estudante, visando um ambiente de ensino mais harmônico e menos custoso (CAZELLA et al., 2012). 
Os chamados SRE's (Sistemas de Recomendação Educacionais) são visualizados no ambiente educacional apoiando-se na proposta de facilitar o trabalho do professor e contribuir com metodologias que visem um aumento na qualidade de ensino, reduzindo a sobrecarga cognitiva ao levar em consideração as preferências e possibilidades individuais (LAISA et al., 2018). Além disso, o trabalho realizado a partir da aplicação de práticas de ensino mais específicas, personalizadas e atualizadas, se mostra eficiente para o estímulo do desenvolvimento de competências e habilidades cognitivas em estudantes, considerando-se que o mesmo seja o elemento central do processo de aprendizagem (SANTOS, 2019).

A respeito, portanto, dessa chance de que os sistemas de recomendação possam ser utilizados para singularizar a trajetória de seus usuários em diferentes aspectos, foi realizada uma revisão sistemática da literatura (RSL), com o objetivo de construir uma visão geral dos estudos que tratam do uso de sistemas de recomendação, como esses sistemas são implementados, com o que podem ser validados e onde são empregados, formando-se, a partir disso, uma base de conhecimento fundamentada na literatura científica visando possibilitar o contato com as ferramentas disponíveis e o auxílio na análise de viabilidade em relação ao desenvolvimento de novas propostas de sistemas de recomendação.

\section{Metodologia de Pesquisa}

Uma revisão sistemática da literatura (RSL) busca sintetizar resultados de pesquisas relacionados a um problema específico (GALVÃO; SWADA; TREVIZAN, 2009). A metodologia de pesquisa utilizada para a condução dessa RSL foi baseada nas diretrizes de Kitchenham e Charters (2007) e de Ferenhof e Fernandes (2016), as quais explicitam a necessidade da definição de questões de pesquisa (subseção 2.1), da estratégia de busca (subseção 2.2) e da seleção dos artigos (subseção 2.3).

\subsection{Questões de pesquisa}

Com o objetivo de se obter conhecimento acerca do estado da arte da implementação de SRE's no Brasil e fornecer um panorama dos estudos apresentados na literatura envolvendo este tema, foram formuladas as seguintes questões de pesquisa (QP) que buscam atender o objetivo proposto: QP1: Como estão sendo implementados os sistemas de recomendação? QP2: Quais os objetivos e aplicações dos sistemas de recomendação na educação? QP3: Quais são os métodos aplicados para a validação do projeto? QP4: Quais os desafios enfrentados no desenvolvimento e na implantação? QP5: Em qual âmbito educacional os sistemas são empregados?

\subsection{Estratégia de busca}

A estratégia de busca utilizada divide-se em duas etapas: na primeira, definem-se as palavras-chave que irão compor a String de busca; em seguida, são definidas as bases bibliográficas, revistas e conferências que serão utilizadas como fonte de estudos. Sabendo que as revistas e fontes trataram sempre de assuntos educacionais, a palavra-chave escolhida e integrante da String de busca foi: recomendação, e para segunda etapa foram selecionadas 10 bases de dados bibliográficas que atenderam aos critérios de disponibilidade dos artigos com resultados trazidos pela pesquisa com a String definida.

As bases bibliográficas utilizadas para a obtenção dos estudos foram: Periódicos Capes; Workshop do Congresso Brasileiro de Informática na Educação (CBIE); Workshop de Desafios da Computação Aplicada à Educação (DESAFIE); Revista Informação e Sociedade (I\&S); Revista Brasileira de Ensino de Ciência e Tecnologia (RBECT); Revista Brasileira de Informática na Educação (RBIE); Revista Novas Tecnologias na Educação (RENOTE); Simpósio Brasileiro de Informática na Educação (SBIE); Scientia Plena; Workshop de Informática na escola (WIE). 
A busca por artigos utilizando a String "recomendação", retornou uma quantidade grande de estudos no Periódicos Capes, portanto, especificamente para essa base, realizou-se a adequação das palavras-chave e consequentemente da String de busca, sendo: ("sistema de recomendação" OR "sistemas de recomendação") AND ("educação" OR "ensino" OR "educacional"). Os últimos acessos às bases de dados foram realizados entre abril e junho de 2021.

\subsection{Seleção dos artigos}

A fim de analisar os estudos obtidos nas pesquisas, foram elaborados critérios de exclusão, que auxiliaram na filtragem dos estudos que tem ligação com o tema proposto. São eles: artigo curto ( $<=3$ páginas); artigo em Inglês; estudo fora do escopo; estudo secundário ou terciário; livro ou dissertação; publicação antes de 2015. Todos os 205 artigos retornados pelos motores de busca foram analisados inicialmente pela leitura do título, resumo, ano de publicação e tipo do documento, possibilitando a seleção criteriosa destes elementos. Após a aplicação dos critérios de exclusão, 46 artigos foram considerados para a extração de dados. A Tabela 1 apresenta o resultado geral das buscas já com a seleção de estudos realizada.

Tabela 1 - Resultado das buscas

\begin{tabular}{l|c|c|c|c|c|c|c|c|c|c|c}
\hline Base de dados & Capes & CBIE & DESAFIE & I\&S & RBECT & RBIE & RENOTE & SBIE & Scientia Plena & WIE \\
\hline \hline Quantidade inicial & 18 & 14 & 1 & 10 & 19 & 19 & 33 & 83 & 5 & 3 \\
Artigos incluidos & 0 & 8 & 0 & 0 & 0 & 8 & 7 & 23 & 0 & 0 \\
\hline
\end{tabular}

É válido ressaltar que 56 dos 159 artigos excluídos, ou seja, aproximadamente $35 \%$, foram rejeitados por terem sido publicados anteriormente ao ano de 2015. A escolha de tal critério se deu, inclusive, pelo fato desta RSL buscar respostas às questões voltadas à utilização de métodos e tecnologias, e, portanto, neste caso, é interessante examinar informações atuais.

Para esta RSL fora empregada uma avaliação de qualidade que auxiliou na extração dos dados e na identificação dos estudos mais completos acerca do tema abordado. Os 46 artigos foram avaliados em relação a um conjunto de 10 critérios de qualidade e as questões utilizadas conforme sugerido por Dermeval et al. (2016), Kitchenham and Charters (2007), Tiwari e Gupta (2015) e Wieringa et al. (2006), podem ser observadas na Tabela 2, bem como a quantidade de artigos que pontuaram em cada quesito. Para cada questão deve-se selecionar uma das três opções que possuem atribuídas a elas uma pontuação, são elas: "'Sim" $=1,0$, "Parcialmente" $=0,5$ e "Não" $=$ 0 .

Tabela 2 - Avaliação de qualidade

\begin{tabular}{|c|c|c|c|}
\hline Questīes & Sim & Par & Nīo \\
\hline Existe um enunciado claro dos objetivos da pesquisa (DERMEVAL et al.. 2016)? & 38 & 7 & 1 \\
\hline A técnica proposta é descrita de forma clara (DERMEVAL et al., 2016)? & 36 & 8 & 2 \\
\hline Os dados de análise foram suficientemente rigorosos (TIWARI; GUPTA, 2015)? & 20 & 16 & 10 \\
\hline Há discussấo suficiente sobre os trabalhos relacionados (TIWARI; GUPTA, 2015)? & 34 & 6 & 6 \\
\hline $\begin{array}{l}\text { Os participantes de estudo ou unidades de observação são descritos adequadamente (KIT- } \\
\text { CHENHAM; CHARTERS, 2007)? }\end{array}$ & 21 & 9 & 16 \\
\hline As limitaçōes desse estudo șo discutidas explicitamente (DERMEVAL et al., 2016)? & 11 & 8 & 27 \\
\hline Há uma discussão sobre os resultados do estudo (DERMEVAL et al., 2016)? & 28 & 12 & 6 \\
\hline As liçōes aprendidas sầo interessantes (TIWARI: GUPTA, 2015)? & 23 & 18 & 5 \\
\hline O artigo é relevante para praticantes (TIWARI; GUPTA, 2015)? & 31 & 14 & 1 \\
\hline É suscetivel a provocar discussä̀o (WIERINGA et al., 2006)? & 33 & 12 & 1 \\
\hline
\end{tabular}

A pontuação de qualidade de um estudo se dá, portanto, pela soma das pontuações das respostas e para esta RSL aqueles que apresentaram uma pontuação menor que 5, não foram considerados para as próximas etapas. Esta RSL, portanto, conta 
com análise aprofundada de 41 estudos. A classificação realizada aumenta a precisão, credibilidade, integridade e relevância dos estudos selecionados.

Salienta-se que os artigos que não atingiram pontuação mínima definida, não possuem qualidade técnica inferior aos demais; a pontuação é referente exclusivamente a relevância dos artigos para esta RSL.

\section{Resultados e Discussões}

O estudo se iniciou com 205 artigos obtidos a partir da busca pela String definida e 41 restaram para análise e extração de dados após a aplicação de critérios de exclusão e qualidade. As referências desta RSL, bem como a sumarização dos artigos que serão identificados pelo ID nas próximas subseções durante a análise de resultados, podem ser vistas na Tabela 3 e na íntegra no endereço $<$ https://bit.ly/3BtSLpQ $>$.

Visando garantir reprodutibilidade, validade e confiabilidade desta RSL, disponibilizou-se as planilhas de extração no seguinte endereço eletrônico: $<$ https://bit.ly/3hP1Lzq $>$.

Tabela 3 - Artigos incluídos na RSL e suas pontuações da avaliação de qualidade

\begin{tabular}{|c|c|c|c|c|c|}
\hline ID & Referência & Pont & ID & Referência & Pont \\
\hline EI & (AGUIAR; FECHINE; COSTA, 2018) & 8.0 & E21 & $\begin{array}{l}\text { (KELLERMANN; SCHMITT; MO- } \\
\text { RAES, 2019) }\end{array}$ & 6.0 \\
\hline E2 & (AGUIAR; FECHINE; COSTA, 2015) & 8.0 & E22 & (MACHIAVELLI et al., 2017) & 7.5 \\
\hline E3 & (ALMEIDA et al., 2016) & 7,0 & E23 & (MARIA; MACEDO; BEHAR, 2016) & 6.0 \\
\hline E4 & (BÓBÓ et al., 2019) & 8.5 & E24 & $\begin{array}{l}\text { (MELO; DANTAS; FERNANDES, } \\
\text { 2017) }\end{array}$ & 9.0 \\
\hline E5 & (BARRÉRE et al., 2020) & 7.5 & E25 & (MENDES et al., 2017) & 6.5 \\
\hline E6 & (BARVINSKI et al., 2017) & 6.0 & E26 & (MIRANDA et al., 2017) & 7,0 \\
\hline E7 & (BEZERRA et al., 2018) & 9.5 & E27 & (MONTEVERDE et al, 2017) & 7.5 \\
\hline E8 & (BORGES et al., 2017) & 6.5 & E28 & $\begin{array}{l}\text { (NEVES; ISHITANI; BRANDĀO, } \\
\text { 2016) }\end{array}$ & 5.5 \\
\hline E9 & $\begin{array}{l}\text { (BREMGARTNER; NETTO; MENE- } \\
\text { ZES, 2015) }\end{array}$ & 7.5 & E29 & (NUNES et al., 2017) & 9.5 \\
\hline E10 & $\begin{array}{l}\text { (BREMGARTNER; NETTO; MENE- } \\
\text { ZES, 2018) }\end{array}$ & 8.0 & E30 & (OLIVEIRA et al., 2018) & 7.5 \\
\hline E11 & $\begin{array}{l}\text { (BREMGARTNER; NETTO; MENE- } \\
\text { ZES, 2016) }\end{array}$ & 5.0 & E31 & (PEREIRA et al., 2015a) & 9.5 \\
\hline E12 & (CARVALHO, 2015) & 9.0 & E32 & (REIS; BARRÉRE, 2015) & 8.0 \\
\hline E13 & (CARVALHO et al., 2016) & 6.5 & E33 & (REZENDE et al., 2018) & 10.0 \\
\hline E14 & $\begin{array}{l}\text { (CASAGRANDE; KOZIMA; WILL- } \\
\text { RICH, 2015) }\end{array}$ & 8.0 & E34 & (ROLIM; FERREIRA; COSTA, 2017) & 9.5 \\
\hline E15 & $\begin{array}{l}\text { (COSTA; JÚNIOR; FERNANDES, } \\
\text { 2019) }\end{array}$ & 5.5 & E35 & (SANTOS; CECHINEL, 2015) & 8.5 \\
\hline E16 & (DANTAS et al., 2018) & 8.5 & E36 & (PEREIRA et al., 2015b) & 6.5 \\
\hline E17 & (FERREIRA et al., 2015) & 5.5 & E37 & (SIMON et al., 2020) & 9.0 \\
\hline E18 & (FERREIRA et al., 2018) & 9.5 & E38 & (STOLL; CURY; MENEZES, 2018) & 8.5 \\
\hline E19 & $\begin{array}{l}\text { (FERREIRA; VASCONCELOS, } \\
2017 \text { ) }\end{array}$ & 7.5 & E39 & (STOLL et al., 2019) & 9.0 \\
\hline E20 & (JÚNIOR; DORÇA, 2018) & 7.0 & $\begin{array}{l}\text { E40 } \\
\text { E41 }\end{array}$ & $\begin{array}{l}\text { (TRINDADE et al., 2020) } \\
\text { (VARGAS et al., 2017) }\end{array}$ & $\begin{array}{l}8.0 \\
8.0\end{array}$ \\
\hline
\end{tabular}

\subsection{Visão geral dos estudos}

Além da apresentação dos resultados de cada pergunta de pesquisa, foram analisadas as características gerais dos artigos incluídos nesta revisão, como ano e local de publicação.

Os artigos selecionados para análise foram publicados no período de 7 anos, entre 2015 e 2021. A Figura 1 apresenta a quantidade de artigos analisados por ano de publicação e aprovados, de acordo com os critérios definidos. 

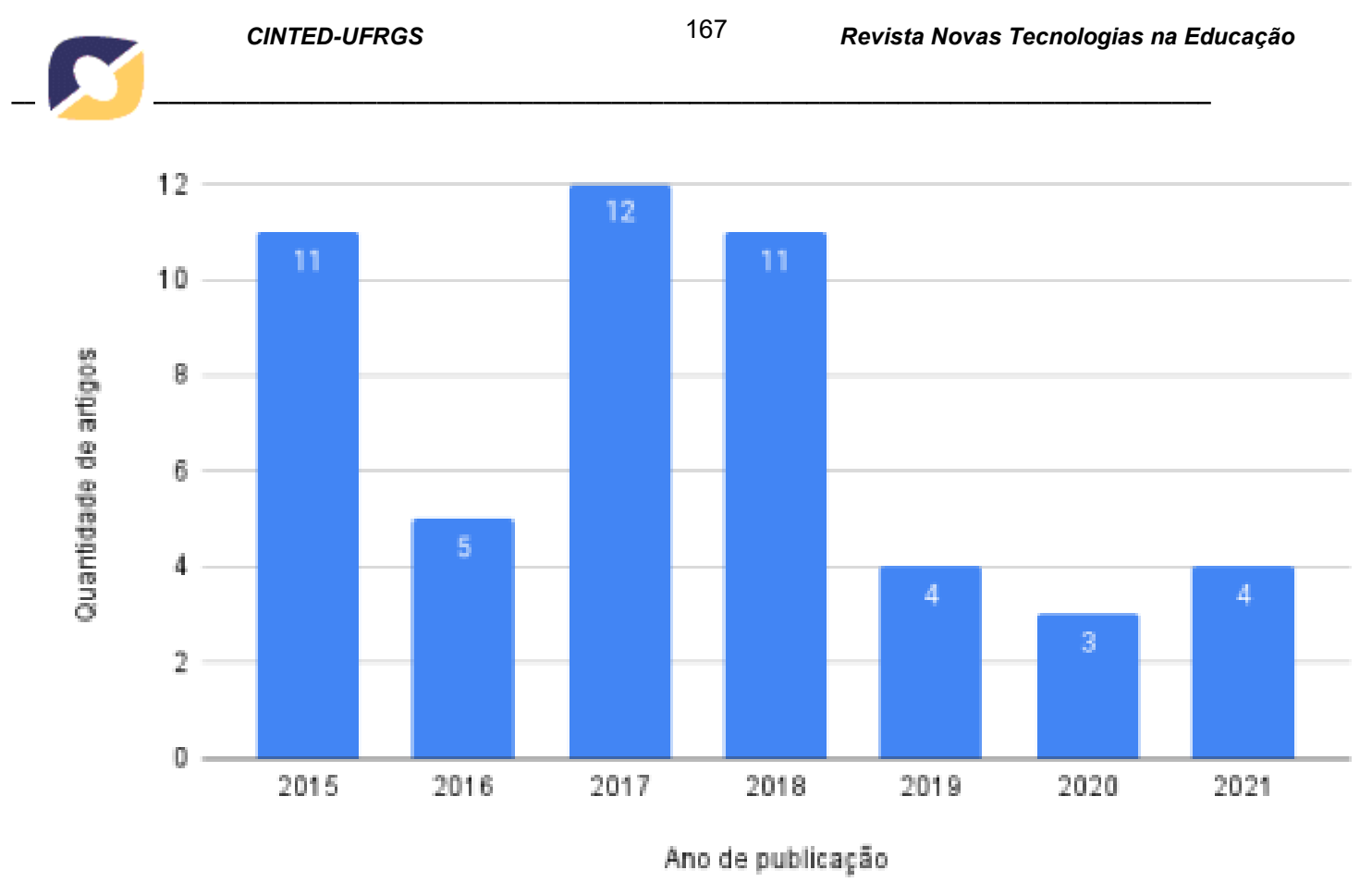

Figura 1 - Quantidade de artigos x Ano de publicação - Pós aplicação de critérios de seleção

Dos 111 trabalhos correspondentes à String de busca e pertencentes ao intervalo definido, somente 46 estavam completamente alinhados aos objetivos deste trabalho. Desses 46 trabalhos, 5 artigos ainda foram excluídos por reprovação na avaliação de qualidade realizada. Os artigos incluídos na extração de dados desta revisão são trabalhos publicados em revistas $(31,71 \%, 13$ artigos $)$, congresso $(17,07 \%, 7$ artigos) e simpósio (51,22\%, 21 artigos), sendo estes: RBIE, CBIE, RENOTE e SBIE. Finalmente, analisou se a distribuição dos artigos por região do Brasil com base em seu vínculo à instituições de ensino. Dentre os 41 artigos selecionados, observou-se a contribuição de autores de 25 instituições. As instituições que se destacaram foram a Universidade Federal de Uberlândia (UFU) e a Universidade Federal do Rio Grande do Sul (UFRGS), ambas com 9 publicações, a Universidade Federal de Juiz de Fora (UFJF), com 7 publicações, a Universidade Federal do Amazonas (UFAM), com 4 publicações e a Universidade Federal do Alagoas (UFAL), com 3 publicações. A Universidade Federal de Campina Grande (UFCG), a Universidade Federal de Pernambuco (UFPE), a Universidade Federal do Espírito Santo (UFES) e o Instituto Federal do Amazonas (IFAM), participaram com 2 artigos cada. As demais instituições tiveram uma publicação cada.

\subsection{Análise das questões de pesquisa}

Analisando os dados referentes às questões de pesquisa envolvidas nesta RSL, para cada uma das questões, descreveu-se e analisou-se os resultados obtidos dos artigos.

\subsubsection{QP1: Como estão sendo implementados os sistemas de recomendação?}

\subsubsection{Padrões de metadados}

Dos estudos selecionados, apenas os de índice E7, E15 e E23 (3 artigos, 7,32\%), não apresentaram informações quanto às tecnologias utilizadas no projeto. Todos os demais citaram ferramentas e métodos específicos. O Felder-Silverman Learning Styles Model (FSLSM) é amplamente utilizado contando com $24,39 \%$ de participação na definição de estilo de aprendizagem (EA) dos estudos de recomendação, sendo utilizado nos artigos E1, E2, E4, E9, E11, E12, E13, E25, E30 e E32. Tal notoriedade se dá, pelo fato de em 1988, Felder e Silverman terem percebido que as pessoas possuem maneiras diferentes de desenvolvimento no que se refere ao recebimento, absorção e compreensão de informações e conhecimentos. São esses os chamados Estilos de Aprendizagem (EA) que, divididos em dimensões, auxiliam, ainda hoje, a construção de uma base para representação de perfil de estudantes em sistemas adaptativos na educação 
(CARVALHO, 2015). Outro termo muito citado nas pesquisas trata-se do padrão Learning Object Metadata (LOM), definido pelo Institute of Electrical and Electronic

Engineers (IEEE) e conhecido como IEEE LOM; um padrão de metadados criado para definir e classificar objetos de aprendizagem (OA) em nove categorias (Geral, Ciclo de vida, Metadado, Técnico, Educacional, Direitos, Relação, Anotação e Classificação) aumentando a eficiência na definição da afinidade entre objetos recomendados e um perfil, e, em sua forma original, tornando possível a replicação dos OA's em diferentes sistemas - o que pode ser resumido nas características Interoperabilidade, Acessibilidade e Durabilidade. Tal padrão aparece em 8 artigos (19,51\%), são eles: E2, E9, E11, E12, E13, E17, E25 e E36. Vale ressaltar que nesta RSL realizada com estudos da literatura nacional, o padrão IEEE LOM recebeu destaque significativo. Nesse contexto, deve-se comentar sobre a existência do OBBA, um padrão de metadados de Objetos de Aprendizagem proposto pela Universidade Federal do Rio Grande do Sul. O destaque ao padrão IEEE LOM pode estar relacionado à sua difusão e consolidação, tendo em vista o maior tempo de uso no cenário tecnológico. Ainda nesse contexto, no ano de 2016, de acordo com Reis e Ferneda (2016), a proposta OBBA não havia sido utilizada em quantidade suficiente para efeitos de comparação, sendo que o universo de metadados dos dois padrões é visto como subutilizado uma vez que poucos dos diversos atributos disponíveis são aplicados em sua totalidade.

\subsubsection{Algoritmos}

Os algoritmos de aprendizado de máquina também aparecem com uma boa frequência nos estudos, visto que estes são aqueles que devem trabalhar toda a lógica das recomendações, seja nas definições de grupos, nas tomadas de decisão ou nas métricas de qualidade, por exemplo. Os mais utilizados nos estudos desta RSL são: K-ésimo vizinho mais próximo (KNN) presente nos estudos E1, E14, E16, E18, E30, E38 e E39 (7 artigos, 17,07\%); Árvore de Decisão em E19, E24, E34, E35, E38 e E39 (6 artigos, 14,63\%); Naive Bayes com aparições nos estudos E18, E30, E34, E35, E38 e E39 (6 artigos, 14,63\%); Perceptron multicamadas (MLP) em E24, E30, E34, E38 e E39 (5 artigos, 12,20\%); J48 em E18, E19, E34 e E35 (4 artigos, 9,76\%); K-Means em E10, E21, E25 e E27 (4 artigos, 9,76\%); Support Vector Machines (SVM) E30, E35, E38 e E39 (4 artigos, 9,76\%). Outros algoritmos deste mesmo grupo foram citados em proporções menores: Rede Bayesiana em E6 e E35 (2 artigos, 4,88\%); Floresta aleatória em E18 (1 artigo, 2,44\%); Algoritmo de maximização de expectativa (EM) em E25 (1 artigo, 2,44\%); AdaBoost em E18 (1 artigo 2,44\%); Self Organizing Map (SOM) E25 (1 artigo, 2,44\%). O pacote de software Weka - que contém uma coleção de algoritmos também de aprendizado de máquina para mineração de dados e desenvolvido em Java encabeça os estudos E10, E25, E27, E34, E35 e E36 (6 artigos, 14,63\%).

\subsubsection{Linguagens}

Notou-se também o uso das tecnologias JAVA, apontada nos artigos E3, E16, E17, E27, E32, E33 e E36 (7 artigos, 17,07\%); MySQL em E3, E8, E22, E32, E33 e E36 (6 artigos, 14,63\%); Python em E4, E20, E26, E30, E38 e E39 (6 artigos, 14,63\%); JADE em E8, E9, E10, E11 e E33 (5 artigos, 12,20\%); Web Ontology Language (OWL) em E13, E17, E20, E33 e E37 (5 artigos, 12,20\%); e XML em E5, E12, E13, E17 e E20 (5 artigos, 12,20\%).

Informações mais detalhadas sobre todos os recursos, inclusive os menos frequentes, podem ser observadas na planilha disponibilizada.

\subsubsection{QP2: Quais os objetivos e aplicações dos sistemas de recomendação na educação?}

Após a leitura dos artigos, foi possível identificar os objetivos e aplicações de cada projeto. Dos 41 artigos, a maioria (28 artigos, 68,29\%) está relacionada à recomendação de OA's para estudantes (E1, E2, E3, E7, E9, E10, E11, E12, E13, E14, E17, E19, E20, E21, E22, E23, E25, E28, E30, E31, E32, E33, E34, E35, E36, E37, 
E40, E41), 5 artigos (12,2\%) estão relacionados à recomendação de ações pedagógicas para professores (E6, E15, E16, E38, E39), 3 artigos (7,32\%) estão relacionados à recomendação de grupos de aprendizagem para estudantes (E18, E21, E27), 3 artigos

$(7,32 \%)$ estão relacionados à recomendação de estratégias pedagógicas para estudantes (E24, E26, E29), 2 artigos (4,88\%) estão relacionados à recomendação de OA's para professores (E5, E8) e apenas 1 artigo $(2,44 \%)$ está relacionado à recomendação de mensagens motivacionais para estudantes.

Os resultados obtidos com a realização desta RSL mostram que uma proporção muito alta dos trabalhos sobre SRE's estão relacionados à aplicação desses sistemas para recomendar OA's para estudantes, com uma diferença bem grande para a segunda aplicação que mais aparece. Também ficou evidente que isso acontece devido à grande e crescente quantidade de conteúdo disponível de forma digital para os estudantes, o que traz uma dificuldade cada vez maior na filtragem de conteúdo mais relevante. Além disso, os demais principais tipos de aplicação também se relacionam de alguma forma ao processo de aprendizagem com foco em benefícios diretos ou indiretos para estudantes, o que mostra que a maior preocupação quando se trata de recomendação em ambientes educacionais é sempre com o objetivo de facilitar esse processo.

\subsubsection{QP3: Quais são os métodos aplicados para a validação do projeto?}

Como método de validação do projeto de SRE descrito, foram mencionadas, entre os estudos analisados: a aplicação de questionários aos usuários (6 artigos, $14,63 \%$ ); a realização de análise experimental (5 artigos, 12,20\%), com exemplos teóricos ou práticos com grupos de usuários predeterminados; o uso de métricas de validação (5 artigos, $12,20 \%)$, como a acurácia e a pureza; a execução de testes (5 artigos, 12,20\%) de usabilidade e com amostragem; estudos de caso ( 3 artigos, 7,32\%); uso de técnicas de validação ( 2 artigos, $4,88 \%$ ), como a $k$-fold cross validation; o uso de sistemas de apoio (2 artigos, 4,88\%); a observação de mudanças nos indicadores do contexto no qual o sistema foi aplicado ( 2 artigos, 4,88\%); realização de simulação empregando-se todas as combinações de cenários possíveis para testagem (1 artigo, $2,44 \%$ ); coleta de dados ao longo do tempo de uso (1 artigo, $2,44 \%$ ); implementação de protótipo para avaliação do sistema de recomendação (1 artigo, 2,44\%); execução do algoritmo (1 artigo, 2,44\%) e comparação dos resultados do algoritmo genérico com os resultados de um algoritmo já empregado (1 artigo, 2,44\%).

Por fim, dos 41 trabalhos, 6 artigos $(14,63 \%)$ não mencionaram a estratégia utilizada para validação do projeto.

A distribuição dos métodos citados está representada na Figura 2.

\subsubsection{QP4: Quais os desafios enfrentados no desenvolvimento e na implantação?}

Dentre os 41 artigos analisados, poucos descreveram os desafios enfrentados no desenvolvimento e na implantação do sistema. Grande parte deles (29 artigos, 70,73\%) não menciona tais aspectos no estudo e não realizou a implantação, o que pode ser um indicador da existência de uma certa dificuldade em se prever ou mensurar os desafios da aplicação antes do evento em si. 


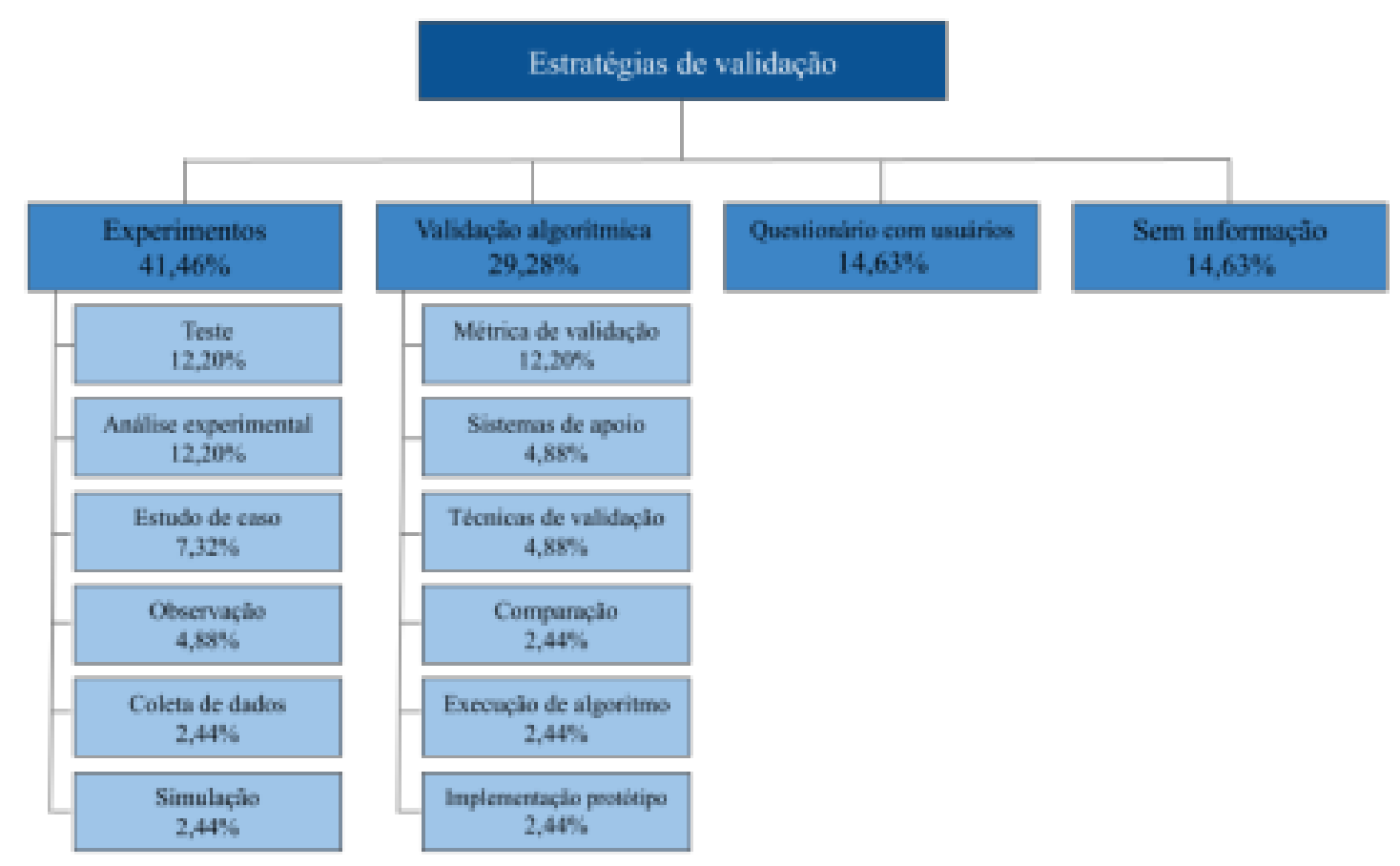

Figura 2 - Estratégias de validação

Por outro lado, entre os pontos indicados pelos artigos que citaram desafios e suas consequências, temos: a falta de evidências dos benefícios de se combinar estilos de aprendizagem com métodos de ensino (E1); resultados diretamente dependentes da quantidade de objetos de aprendizagem disponibilizados (E12, E18) e, por consequente, a dependência da ativa participação dos usuários (E21); a alta necessidade de atenção com a acuracidade de dados obtidos por meio de ambientes virtuais (E15) conscientizando-se da existência de ruídos em processos de identificação através de questionários (E18), como a dificuldade em manter um padrão de anotações feitas pelos colaboradores da pesquisa (E28); a dificuldade de obtenção de bases para treinamento dos classificadores (E18); a definição de variáveis mais próximas da realidade para uso em cenário de simulação (E20); a necessidade de conhecimento prévio sobre os objetos de aprendizagem para uso da ferramenta - por parte dos usuários - e suas variações (E33) o que fornece certa complexidade ao uso de SRE's (E37), exigindo a adaptação do formato de alguns dados após a primeira etapa de uso (E35, E37).

Além disso, há de se considerar a quantidade de dados para trabalho, uma vez que o grande volume de conteúdos exigindo a busca por diferentes conceitos pode apresentar ao usuário uma filtragem desorganizada ou pouco compreensível, devolvendo ao mesmo o papel de selecionar manualmente os materiais de interesse (E40), e a escassez de dados e itens pode gerar um problema chamado cold-start, reconhecido como um problema dos sistemas de recomendação, conforme indicado pelo estudo E7, onde a falta de informações pode impossibilitar a recomendação.

\subsubsection{QP5: Em qual âmbito educacional os sistemas são empregados?}

Analisando-se os resultados obtidos pela realização desta revisão, pode-se concluir, respondendo à questão QP5, que os estudos relacionados a sistemas de recomendação na educação têm sido majoritariamente aplicados ao ensino superior - considerando-se todo o contexto universitário: alunos de graduação, pós-graduação e professores - com integração a ambientes de aprendizagem (24 artigos, 58,54\%) compreendendo os estudos E2, E4, E5, E6, E7, E10, E11, E15, E16, E18, E19, E21, E22, E23, E24, E25, E26, E27, E30, E33, E34, E37, E38 e E39, o que evidencia a recorrência do tema dentro de tal segmento. 
Verificou-se também 6 artigos $(14,63 \%)$ que foram empregados em pesquisa geral, destinando-se a quaisquer segmentos que façam uso de tecnologias voltadas para a educação, como cursos profissionalizantes, compreendendo os estudos E31, E32, E35, E36, E40 e E9, e 11 artigos (26,83\%) nos quais não se definiu o nível educacional (E1, E3, E8, E12, E13, E14, E17, E20, E28, E29 e E41), sendo que em um deles a solução foi somente objeto de estudo algorítmico.

Apesar de a grande maioria ter sido primeiramente empregada no Ensino Superior, entre os 24 artigos, 7 estudos (E4, E19, E22, E33, E34, E37 e E38) indicaram uma abertura para aplicação das ferramentas em diferentes contextos objetivando a exploração e a complementação de recursos, o que evidencia a importância de que se vislumbra o estudo e aplicação desse tema nos demais níveis educacionais.

\section{Ameaças à validade}

Primeiramente, foram selecionados somente artigos publicados na língua portuguesa. Além disso, apesar dos diversos critérios utilizados para a extração de dados, o risco de perda de dados importantes ainda existe, tendo em vista o julgamento subjetivo feito pelos autores acerca da falta de clareza das informações e relação do estudo com os objetivos da presente revisão, ainda que se tenha tido cuidado em abstrair-se de posições pessoais. Tal abstração realizou-se com o embasamento em estudos anteriores disponibilizados e revisados, técnicas já utilizadas e recomendadas no meio acadêmico e a discussão entre os autores para redução deste impacto.

\section{Conclusão}

De acordo com a RSL realizada, conclui-se que o interesse pela aplicação de melhorias para o ambiente acadêmico em relação às recomendações de conteúdos e de características de personalização vem sendo demonstrado em diversos estudos da área de tecnologia.

A análise dos trabalhos elucidou as tecnologias mais utilizadas para implantação de SRE's, como o modelo FSLSM e o padrão de metadados IEEE-LOM, as técnicas de validação de projetos mais frequentes e as dificuldades encontradas em etapas como o planejamento e o desenvolvimento.

Nesse sentido, é possível visualizar que se aborda pouco sobre a aplicação das propostas, da implementação dos projetos, e muito sobre o planejamento para o desenvolvimento de sistemas. Portanto, a baixa proximidade à realidade - visto que são sistemas hipotéticos em sua maioria - no que se trata das vantagens e desvantagens, pontos positivos ou negativos, pode ser observada como um problema geral.

Finalmente, como resposta para o objetivo de construção de uma visão geral e embasamento para a criação de uma proposta de SRE, é possível concluir que, ainda que sejam diversas as tecnologias disponíveis para a elaboração de um sistema de recomendação, é importante apoiar-se nas contribuições realizadas considerando-se as especificidades dos projetos e aprofundando-se no conhecimento de seus recursos, como os padrões de metadados, por exemplo, pois, ainda que amplamente utilizados ou recentemente elaborados e explorados, há diferentes abordagens que podem ser feitas acerca de seus artifícios.

\section{Referências}

CARVALHO, V. Uma abordagem para recuperação e recomendação automática, dinâmica e não determinística de objetos de aprendizagem baseada em estilos de aprendizagem, em metadados no padrão ieee lom e em elementos de web semântica. In:Anais dos Workshops do Congresso Brasileiro de Informática na Educação. [S.1.: 
s.n.], 2015. v. 4, n. 1, p. 135.

CAZELLA, S. C. et al. Desenvolvendo um sistema de recomendação de objetos de aprendizagem baseado em competências para a educação: relato de experiências. In:Brazilian Symposium on Computers in Education (Simpósio Brasileiro de Informática na Educação-SBIE). [S.1.: s.n.], 2012. v. 23, n. 1.

DERMEVAL, D. et al. Applications of ontologies in requirements engineering: $a$ systematic review of the literature. Requirements Engineering, Springer, v. 21, n. 4, p. 405-437, 2016.

FERENHOF, Helio Aisenberg; FERNANDES, Roberto Fabiano. Desmistificando a revisão de literatura como base para redação científica: método SSF. Revista ACB, v. 21, n. 3, p. 550-563, 2016.

GALVÃO, C. M.; SAWADA, N. O.; TREVISAN, M. A. Revisão sistemática: recurso que proporciona a incorporação das evidências na prática da enfermagem. Rev Lat Am Enferm. 2004; 12 (3): 549-56. Rev Esc Enferm USP, v. 43, n. 2, p. 465-71, 2009.

KITCHENHAM, B. A.; CHARTERS, S. Guidelines for performing Systematic Literature Reviews in Software Engineering. [S 1], 2007. Disponível em: $<$ https://www.elsevier.com-/data/promismisc/525444systematicreviewsguide.pdf $>$. Acesso em: 15 abr. 2021.

REIS, Marcia Cristina dos; FERNEDA, Edberto. Panorama sobre a utilização dos padrões de metadados IEEE LOM e OBAA em repositórios educacionais brasileiros. IV Seminário em Ciência da Informação: Fenômenos Emergentes na Ciência da Informação, Londrina, p. 1141-1156, ago. 2016. Disponível em:

http://www.uel.br/eventos/cinf/index.php/secin2016/secin2016/paper/viewFile/312/201. Acesso em: 11 dez. 2021.

SANTOS, Elisângela Ribas dos. Aprendizagem adaptativa por meio da experiência de aprendizagem mediada. 2019. 145 f. Tese (Doutorado) - Curso de Pós-Graduação em Educação em Ciências e Matemática, Pontifícia Universidade Católica do Rio Grande do Sul, Porto Alegre, 2019. Disponível em:

http://tede2.pucrs.br/tede2/bitstream/tede/8858/2/TeseElisangelaRibas-homologada.pdf. Acesso em: 11 dez. 2021.

LAISA, J. et al. Uma revisão sistemática da literatura sobre sistemas de recomendação educacional. Anais do Computer on the Beach, p. 751-760, 2018.

TIWARI, S.; GUPTA, A. A systematic literature review of use case specifications research Information and Software Technology, v 67, p . 128-158, 2015. Disponível em: $<$ https://www-.sciencedirect.com/science/article/pii/S0950584915001081>. Acesso em: 15.04.2021.

WIERINGA, R. et al. Requirements engineering paper classification and evaluation criteria: a proposal and a discussion. Requirements engineering, Springer, v. 11, n. 1, p. 102-107, 2006. 